\title{
Results from the annual modulation analysis of the XMASS-I dark matter data
}

\author{
Katsuki Hiraide ${ }^{* 1,2}$ on behalf of the XMASS Collaboration \\ ${ }^{1}$ Kamioka Observatory, Institute for Cosmic Ray Research, the University of Tokyo, \\ Higashi-Mozumi, Kamioka, Hida, Gifu, 506-1205, Japan \\ ${ }^{2}$ Kavli Institute for the Physics and Mathematics of the Universe (WPI), the University of Tokyo, \\ Kashiwa, Chiba, 277-8582, Japan \\ E-mail: hiraide@km.icrr.u-tokyo.ac.jp
}

The annual modulation of the event rate in direct dark matter detection experiments would be a strong signature of dark matter. XMASS-I, the first phase of the XMASS project, is designed to detect dark matter using $832 \mathrm{~kg}$ of liquid xenon at Kamioka in Japan. We have continuously collected data since November 2013. In this paper, we present preliminary results from the annual modulation analysis using our 359.2 live days of data collected with the XMASS-I detector.

The 34th International Cosmic Ray Conference,

30 July- 6 August, 2015

The Hague, The Netherlands

\footnotetext{
* Speaker.
} 


\section{Introduction}

Although there are substantial evidences for the existence of dark matter in the Universe from its gravitational effects, the identity of dark matter is still not known. Among the possible dark matter candidates, Weakly Interacting Massive Particles (WIMPs) can be detected directly through observation of nuclear recoils produced in their elastic scattering interactions with detector nuclei. The count rate of dark matter signal is expected to modulate annually due to the relative motion of the Earth around the Sun. The dark matter flux at the detector becomes maximal in June and minimal in December. The annual modulation would be a strong signature for dark matter. The DAMA/LIBRA experiment claims evidence for such a modulating signal [1] and the CoGeNT Collaboration reported a preference for an annual modulation [2]. However, the CDMS II experiment has performed an annual modulation search, finding no evidence for modulation [3].

The XMASS project is designed to detect dark matter, neutrinoless double beta decay, and ${ }^{7} \mathrm{Be} / \mathrm{pp}$ solar neutrinos using highly-purified liquid xenon scintillator in an ultra-low radioactivity environment [4]. As the first stage of the XMASS project (XMASS-I), a detector focusing mainly on direct dark matter search was constructed and commissioning data was taken from December 2010 to May 2012. In order to reduce the backgrounds, detector refurbishment was conducted. After a year of the detector refurbishment, data-taking resumed in November 2013. Owing to its large target mass and low energy threshold, the XMASS-I detector has an advantage in searching for dark matter from annual modulation of the event rate. Moreover, XMASS is sensitive to not only nuclear recoils but also $e / \gamma$ events. In this paper, we present results from the annual modulation analysis using the data collected with the XMASS-I detector after refurbishment.

\section{XMASS-I detector and calibrations}

The XMASS-I detector is a single phase liquid xenon scintillator detector located underground (2700 m water equivalent) at the Kamioka Observatory in Japan. It contains $832 \mathrm{~kg}$ of liquid xenon in an active region. The volume is viewed by 630 hexagonal and 12 cylindrical Hamamatsu R10789 photomultiplier tubes (PMTs) arranged on an $80 \mathrm{~cm}$ diameter pentakis-dodecahedron support structure. A total photocathode coverage of more than $62 \%$ is achieved. The spherical arrays of PMTs are arranged in a double wall vessel made of oxygen free high conductivity (OFHC) copper. In order to shield the liquid xenon detector from external gammas, neutrons, and muon-induced backgrounds, the copper vessel was placed at the center of a $\phi 10 \mathrm{~m} \times 10.5 \mathrm{~m}$ cylindrical tank filled with pure water. The water tank is equipped with 72 Hamamatsu R3600 20-inch PMTs to provide both an active muon veto and passive shielding against these backgrounds. XMASS-I is the first direct detection dark matter experiment equipped with such an active water Cherenkov shield. The liquid xenon and water Cherenkov detectors are hence called an Inner Detector (ID) and an Outer Detector (OD), respectively. Data acquisition is triggered if four or more PMTs have more than 0.2 photoelectron (PE) within 200 ns. More details are described in Ref. [5].

The gain of each PMT is continuously monitored with signals generated by a blue LED embedded on the inner surface of the ID PMT holder and is corrected for using the LED calibration data. Energy calibrations were performed by inserting thin cylindrical calibration sources containing either of ${ }^{55} \mathrm{Fe},{ }^{57} \mathrm{Co},{ }^{109} \mathrm{Cd}$, or ${ }^{241} \mathrm{Am}$ directly into the target volume. In order to monitor $\mathrm{PE}$ 


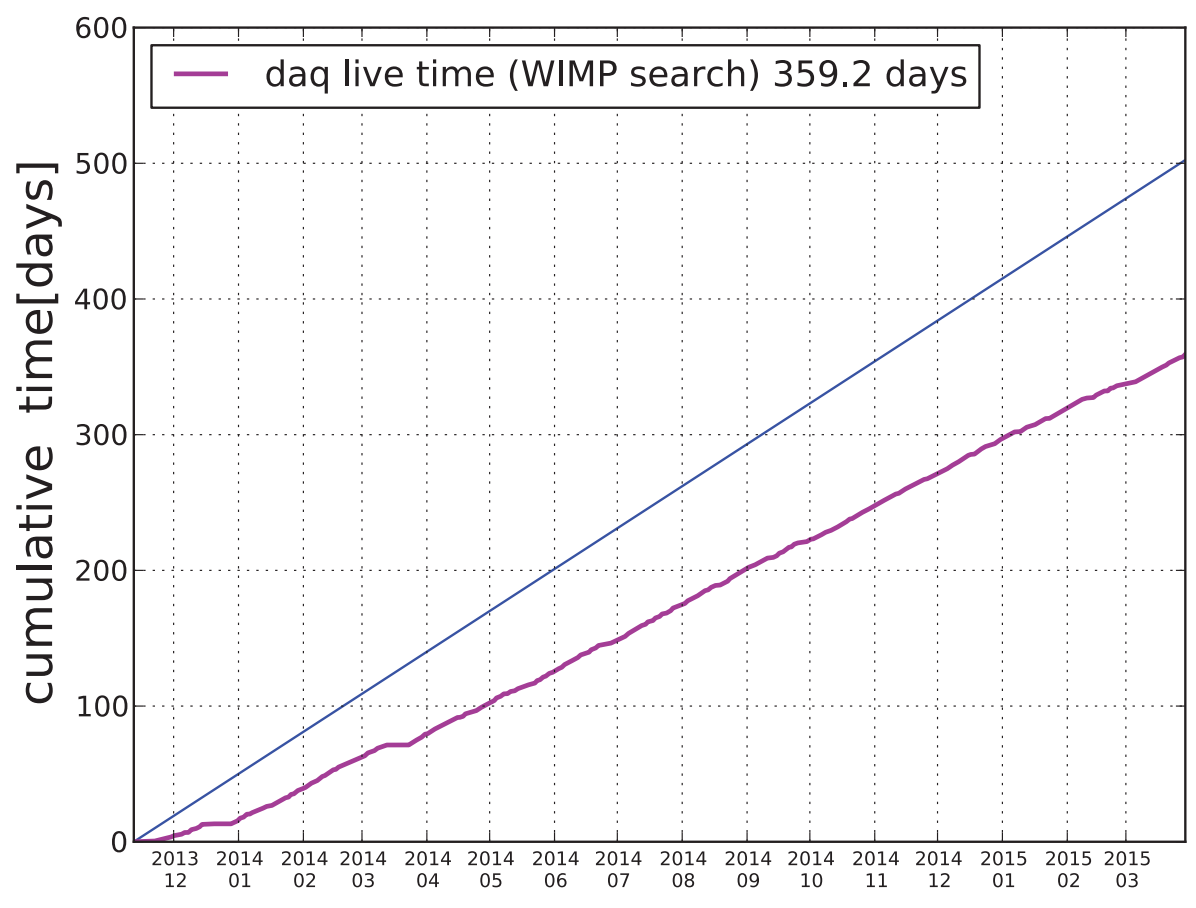

Figure 1: History of the accumulated livetime after data quality cuts for annual modulation analysis. The blue straight line indicates the total elapsed days.

yield and optical properties of the liquid xenon, the detector is calibrated regularly with the ${ }^{57} \mathrm{Co}$ source inserted and placed at various positions along the central vertical axis of the detector. Relative time offset of each PMT signal is monitored and corrected for by the data taken with the ${ }^{57} \mathrm{Co}$ source at the center of the detector volume.

\section{Data set and event selection}

The data used for this analysis was collected between November 20, 2013 and March 29, 2015. We selected periods of operation with a stable temperature and pressure. Periods of operation with excessive PMT noise or abnormal trigger rates were removed. Figure 1 shows the history of the accumulated livetime. The total effective livetime becomes 359.2 days after the data quality cuts mentioned above.

A series of simple event selections is applied to the data set. We select events triggered only by the liquid-xenon detector. In order to remove events caused by afterpulses of PMTs due to bright events, events that occurred within $10 \mathrm{~ms}$ of the previous event are rejected. Events whose timing distribution has an RMS greater than 100 ns are also removed. In order to remove Cherenkov events originated from ${ }^{40} \mathrm{~K}$ in the PMT photocathode, distribution of hits' timing in each event is used. Events with more than $60 \%$ of their PMT hits occurring within the first 20 ns of the event window are removed as Cherenkov-like. Finally, in order to remove events occurred in front of PMT, we search for a hit whose number of PEs is maximal among all hits. Events with a large ratio of the maximum PEs in a PMT to the total PEs in the detector are removed. The cut position 


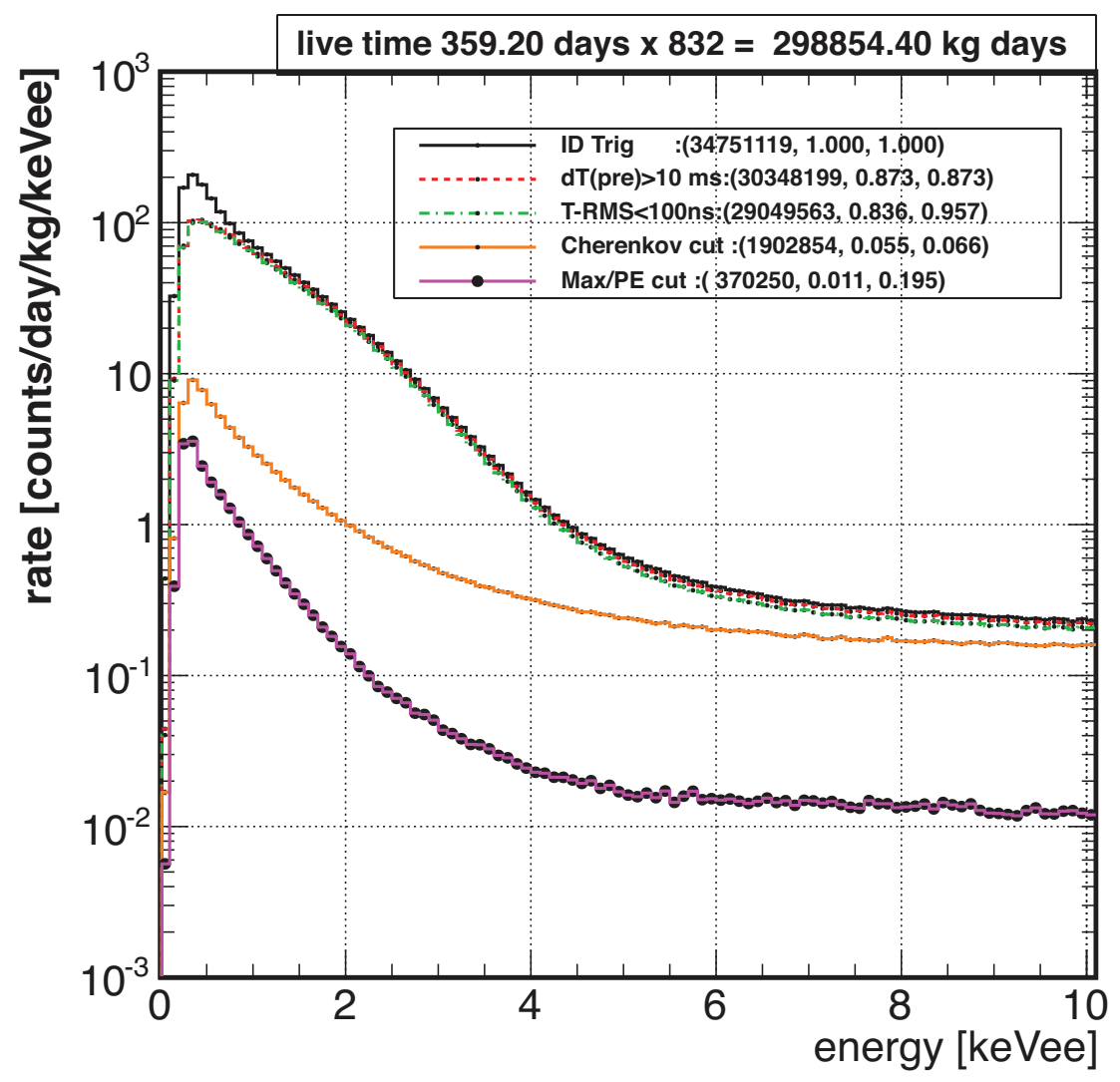

Figure 2: Energy spectra of the observed events after each reduction step for our 359.2 live days of data. From top to bottom, the observed energy spectrum after the inner detector trigger selection, the event time separation cut, the hits' timing RMS cut, the Cherenkov cut, and the maximum PE ratio cut.

was determined so that it contains 50\% of the simulated WIMP signal distributed uniformly in the detector. Figure 2 shows energy spectra of the observed events after each reduction step for our 359.2 live days of data. The energy spectrum and cut efficiency as a function of energy after the same event selection for the simulated $20 \mathrm{GeV} / \mathrm{c}^{2}$ WIMPs are shown in Figure 3.

\section{Detector stability and systematic uncertainty}

Figure 4 (top) shows the stability of the observed PE yield monitored with the ${ }^{57} \mathrm{Co} 122 \mathrm{keV}$ $\gamma$-ray source. It experienced a gradual change from the beginning of the run and a sudden drop at a power failure in August 2014. The stability of optical properties of liquid xenon such as absorption length, scattering length, and the relative intrinsic scintillation light yield are also extracted from the ${ }^{57} \mathrm{Co}$ calibration data as shown in the figure. The PE yield change can be explained by the change of absorption length in liquid xenon.

Since the change of absorption length in liquid xenon affects the cut efficiency, we evaluate the relative change of the cut efficiency using the MC simulation. Figure 5 shows the relative cut efficiency as a function of absorption length in liquid xenon for $0.5-1 \mathrm{keV}_{e e}(\mathrm{left})$ and $1-5 \mathrm{keV}_{e e}$ 

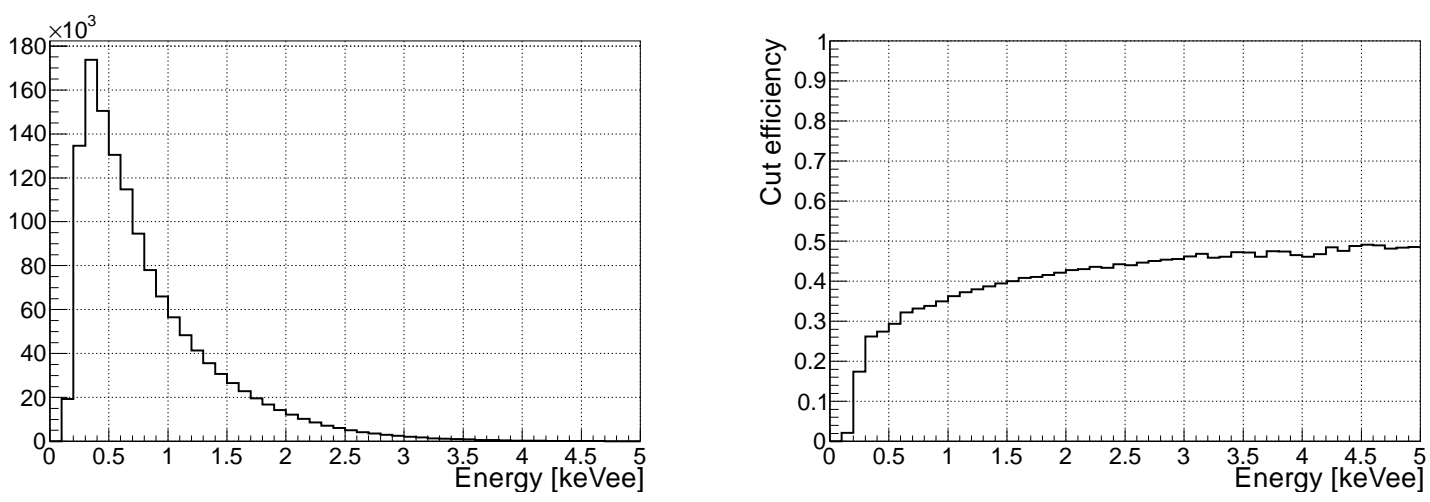

Figure 3: Energy spectrum (left) and cut efficiency as a function of energy (right) after all cuts for the simulated $20 \mathrm{GeV} / c^{2}$ WIMPs.

(right). The central lines are used for data correction, and the colored bands represent the systematic uncertainty due to the position dependence of the detector response. It is a dominant systematic error in this analysis.

\section{Annual modulation analysis}

We perform our analysis on the annual modulation of the event rate using binned chi-square. The dataset is divided into time bins with roughly 10 days of livetime, and each of them is divided into energy bins with a $0.1 \mathrm{keV}_{e e}$ width up to $5 \mathrm{keV}_{e e}$. Our energy threshold for analysis is set to $0.5 \mathrm{keV}_{e e}$. Two independent analyses are performed with different treatment for systematic uncertainty.

The method- 1 introduces nuisance parameters $\alpha_{i}$ and the $\chi^{2}$ is defined as

$$
\chi_{\text {method } 1}^{2}=\sum_{i}^{E-\text { bins }}\left(\sum_{j}^{t-\text { bins }} \frac{\left(R_{i, j}^{\text {obs }}-R_{i, j}^{\text {pred }}-\alpha_{i} K_{i, j}\right)^{2}}{\sigma(\text { stat })_{i, j}^{2}}+\alpha_{i}^{2}\right)
$$

where $R_{i, j}^{\mathrm{obs}}, R_{i, j}^{\mathrm{pred}}$, and $\sigma(\mathrm{stat})_{i, j}$ are the observed and predicted event rate, and the statistical error in the $i$-th energy and $j$-th time bin, respectively. $K_{i, j}$ represents the $1 \sigma$ systematic error on the predicted event rate.

The method- 2 uses a covariance matrix to include effects of systematic error, and the $\chi^{2}$ is defined as

$$
\chi_{\text {method2 }}^{2}=\sum_{i, j}^{E t-\text { bins }}\left(R_{i}^{\text {obs }}-R_{i}^{\text {pred }}\right)\left(V_{\text {stat }}+V_{\text {sys }}\right)_{i j}^{-1}\left(R_{j}^{\text {obs }}-R_{j}^{\text {pred }}\right)
$$

where $R_{i(j)}^{\mathrm{obs}}$ and $R_{i(j)}^{\mathrm{pred}}$ are the observed and predicted event rate in the $i$-th $(j$-th) 2-dimensional $E$ - $t$ bin. $V_{\text {stat }}$ represents the statistical error and $V_{\text {sys }}$ is the covariance matrix for systematic uncertainties.

In order to extract annual modulation in a model independent way, we parameterize the prediction as

$$
R^{\mathrm{pred}}\left(E_{i}, t_{j}\right)=C_{i}+A_{i} \cos 2 \pi\left(t_{j}-t_{0}\right) / T
$$




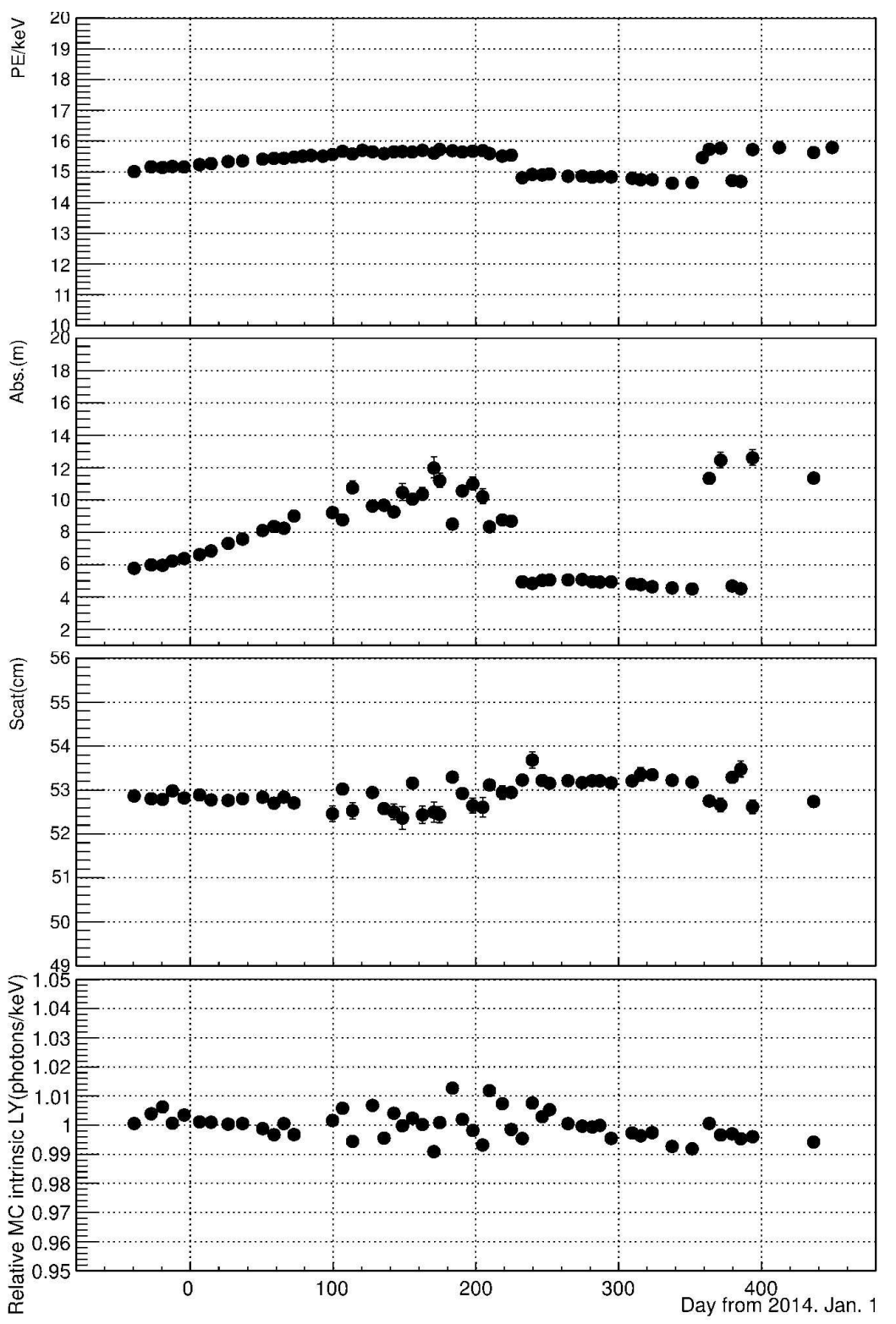

Figure 4: Stability of the observed photoelectron yield, absorption length, scattering length, and relative intrinsic scintillation light yield of liquid xenon.

where $C_{i}$ and $A_{i}$ are the unmodulated event rate and the modulated amplitude to be determined by the fit in the $i$-th energy bin. $T$ and $t_{0}$ are the oscillation period and phase, respectively. In case that we assume WIMP dark matter, the parameterization is changed as

$$
R^{\mathrm{pred}}\left(E_{i}, t_{j}\right)=C_{i}+\sigma_{\chi N} \times A\left(m_{\chi}, E_{i}\right) \cos 2 \pi\left(t_{j}-t_{0}\right) / T
$$

where $\sigma_{\chi N}$ is the WIMP-nucleon cross section, and $A\left(m_{\chi}, E_{i}\right)$ is the modulation amplitude in the $i$-th energy bin depending on the WIMP mass $m_{\chi}$. 

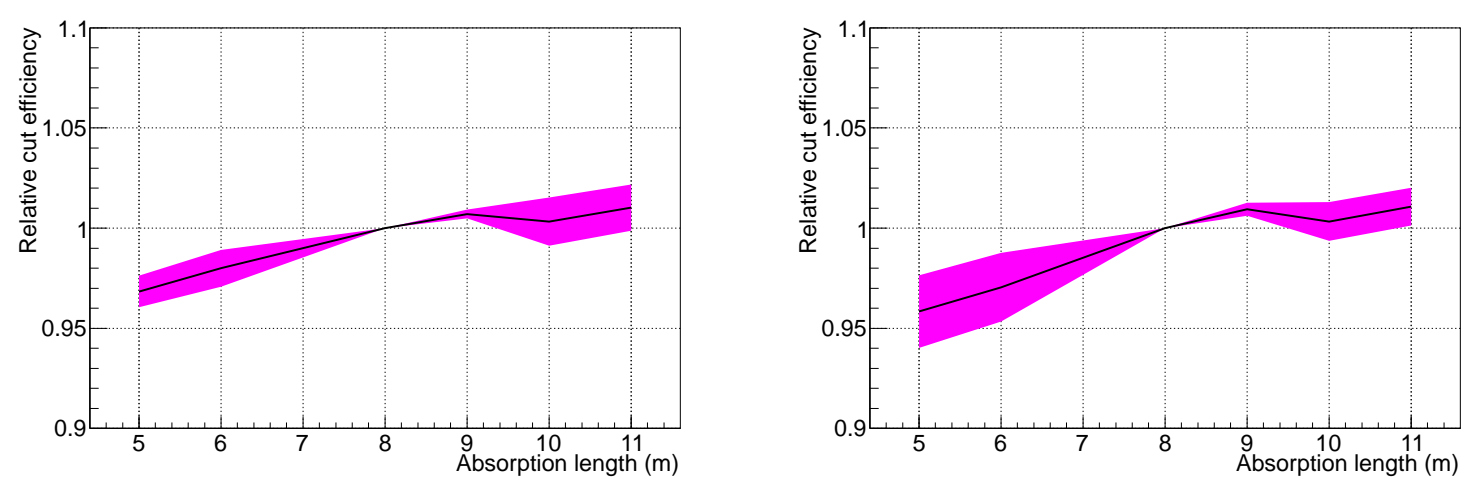

Figure 5: Relative cut efficiency as a function of absorption length in liquid xenon for 0.5-1 $\mathrm{keV}_{e e}$ (left) and 1-5 $\mathrm{keV}_{e e}$ (right). The relative cut efficiency is normalized to 1 for absorption length of $8 \mathrm{~m}$. The solid lines are the central values used for data correction, and the colored bands represent the systematic uncertainty.

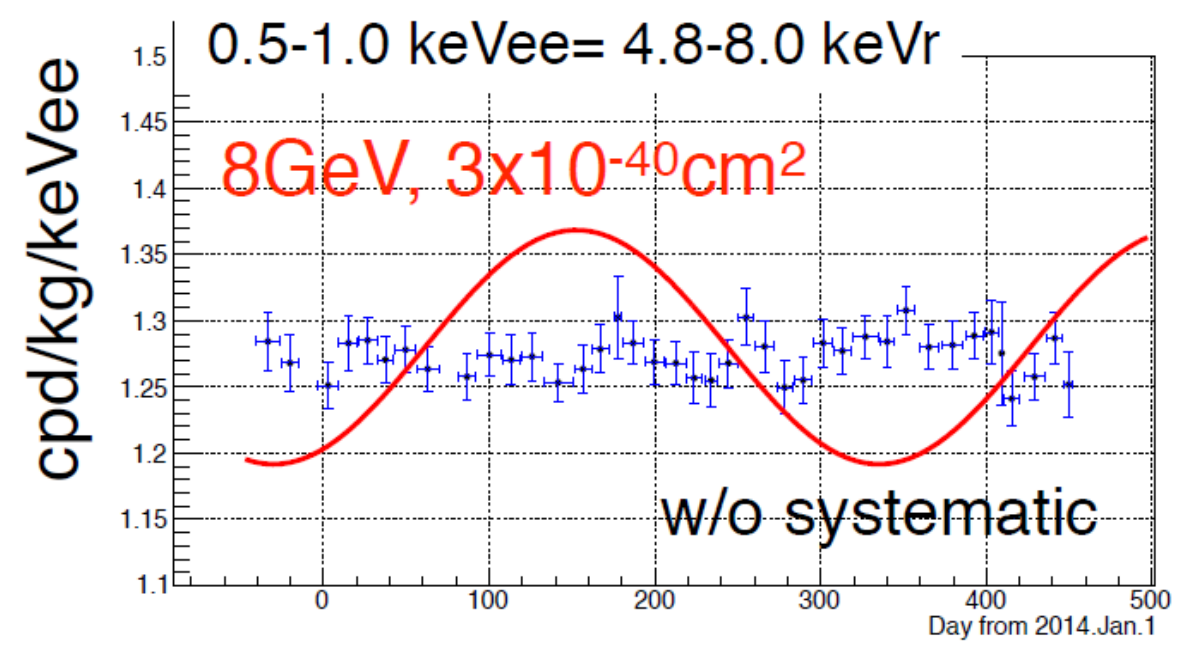

Figure 6: Observed count rate as a function of time in the $0.5-1.0 \mathrm{keV}_{e e}$ energy interval which corresponds to $4.8-8.0 \mathrm{keV}$ nuclear recoil energies. The red curve is the expected annual modulation assuming $8 \mathrm{GeV} / c^{2}$ WIMP with the WIMP-nucleon cross section of $3 \times 10^{-40} \mathrm{~cm}^{2}$.

Figure 6 shows the observed count rate after cuts as a function of time in the $0.5-1.0 \mathrm{keV}_{e e}$ energy interval. The energy region corresponds to $4.8-8.0 \mathrm{keV}$ nuclear recoil energies. Our data is overlaid with the expected annual modulation assuming $8 \mathrm{GeV} / \mathrm{c}^{2}$ WIMP with the WIMP-nucleon cross section of $3 \times 10^{-40} \mathrm{~cm}^{2}$. WIMPs are assumed to be distributed in an isothermal halo with $v_{0}$ $=220 \mathrm{~km} / \mathrm{s}$, a galactic escape velocity of $v_{\mathrm{esc}}=650 \mathrm{~km} / \mathrm{s}$, and an average density of $0.3 \mathrm{GeV} / \mathrm{cm}^{3}$.

Our expected sensitivity to WIMP dark matter is shown in Figure 7. The sensitivity curve covers all the DAMA's allowed region and thus XMASS has a high sensitivity to the annual modulation claimed by the DAMA/LIBRA group. The preliminary $90 \%$ confidence level (CL) upper limit for $14 \mathrm{GeV} / \mathrm{c}^{2}$ WIMP is also shown in the figure. We are finalizing systematic error evaluation and the results will come soon. 


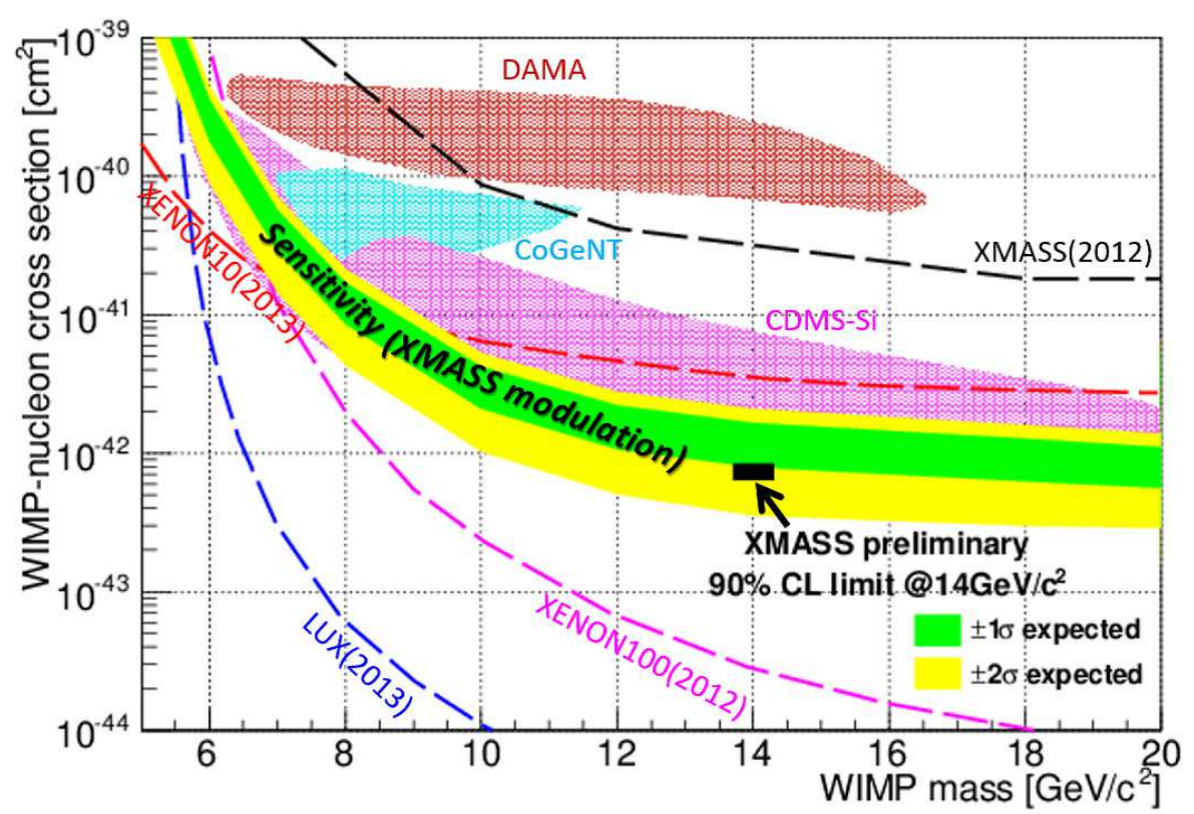

Figure 7: Our expected sensitivity to WIMP dark matter. The preliminary $90 \%$ confidence level upper limit for $14 \mathrm{GeV} / \mathrm{c}^{2}$ WIMP is also shown.

\section{Conclusions}

The XMASS project is designed for multiple physics goals using liquid xenon scintillator. As the first stage of the project, the detector with $832 \mathrm{~kg}$ of liquid xenon was constructed. We performed an annual modulation analysis using our 359.2 live days of data. XMASS has a high sensitivity to the annual modulation claimed by the DAMA/LIBRA group. The results from our one-year data will come soon.

\section{References}

[1] R. Bernabei et al., Eur. Phys. J. C 56, 333 (2008);

R. Bernabei et al., Eur. Phys. J. C 67, 39 (2010).

[2] C. E. Aalseth et al. (CoGeNT Collaboration), Phys. Rev. Lett. 107 (2011) 141301;

C. E. Aalseth et al. (CoGeNT Collaboration), arXiv:1401.3295 [astro-ph.CO].

[3] Z. Ahmed et al. (CDMS II Collaboration), arXiv:1203.1309 [astro-ph.CO].

[4] Y. Suzuki et al., hep-ph/0008296.

[5] K. Abe et al. (XMASS Collaboration), Nucl. Instrum. Meth. A 716 (2013) 78. 